M. Nisio

Nagoya Math. J.

Vol. 52 (1973), 1-30

\title{
ON STOCHASTIC OPTIMAL CONTROL LAWS
}

\author{
MAKIKO NISIO
}

§ 1. Introduction. Let us begin by recalling the existence of optimal controls for a class of stochastic differential equations

$$
d X(t)=\beta(t, X(s), s \leq t, U(t)) d B(t)+\alpha(t, X(s), s \leq t, U(t)) d t, t \geq 0,
$$

with given initial condition $X(0)=x$, where $B$ is an $n$-dimensional Brownian motion and the control $U$ is a stochastic process. As admissible controls, let us allow all non-anticipative process $U(t)$ $=\left(U_{1}(t), \cdots U_{m}(t)\right) \in \Gamma$ where $\Gamma$ is a compact subset of $\boldsymbol{R}^{m}$. We call $\Gamma$ a control region. Assume that the matrix valued functional $\beta$ and the $n$-vector valued $\alpha$ satisfy a Lipscitz condition in $X$ and some growth condition. Then we have a unique solution $X^{U}$ for an admissible control $U$.

We shall consider the minimization problem for the expectation of cost functional $\Phi\left(X^{U}, U\right)$. If $\beta$ does not depend on $U$ and $\alpha$ is linear in $U$, i.e

$$
\alpha_{i}(t f u)=\sum_{j=1}^{m} \alpha_{i j}(t f) u_{j} .
$$

Fleming and Nisio [4] consider the existence of an optimal control $U_{0}$, (open loop control), in the case where $\Phi(X, U)$ is non-negative and lower semi-continuous on $X$ and $V(t)=\int^{t} U(s) d s$. But in many problems of controls, we would like to minimize $E \Phi\left(X^{U}, U\right)$ subject the condition that the control $U(t)$, selected at time $t$, should depend only on the observed data up to time $t$. Let us suppose that the system $X$ of (1.1) is completely observable. Thus an admissible control will be a function $u ;[0 \infty) \times C_{n} \rightarrow \Gamma$, which satisfies the non-anticipative condition. If $\beta$ $\equiv 1$ and $\alpha(t f \Gamma)$ is convex (Roxin's condition), then Beneš [2] proved the existence of an optimal control $u_{0}$, (control based on a complete

Received March 7, 1973. 
observation), in the case where the cost functional $\Phi$ is given by an integral form.

We shall remark, in $\S 5$, that an optimal control based on a complete observation turns out to an optimal open loop control under some conditions. This means that the synthesis problem is decided, i.e. an optimal open loop control will be determined as a function of data of the system $X$. In $\S \S 3 \sim 5$, we assume that $\beta$ does not depend on $U$. The existence of an optimal open loop control will be proved under the Roxin's condition, (A 6) and (A 7), in $\S 3$. Moreover when $\beta$ is uniformly positive definite, we shall consider the correspondence between the laws of response of open loop controls and controls based on a complete observation. Namely, let $\mathscr{P}$ and $Q$ be set of all laws of response of open loop controls and controls based on a complete observation respectively. Then we show that $\mathscr{P}=Q$, in $\S 5$. When $\beta$ depends on $U$, the existence of optimal controls and the synthesis problem will be discussed in $\S 6$. In $\S 7$, we discuss controls of diffusion type processes. We shall sketch the Krylov's work [9], i.e. when the cost functional $\Phi$ is given by an integral form, an optimal control is attained by a Markovian policy, under some conditions. But, when $\Phi$ is not an integral form, we have a little counter example.

Let us now introduce some preliminary definitions and notations.

Given a stochastic process $X(t), t \geq 0, \mathfrak{B}_{t}(X)$ denotes the least $\sigma$ algebra generated by $\{X(s), s \leq t\}$.

The $n$-dimensional Brownian motion is denoted by $B(t)=\left(B_{1}(t)\right.$, $\left.\cdots B_{n}(t)\right), t \geq 0$, and we normalize it by $B(0)=0 . \quad \mathfrak{B}_{t v}(d B)$ denotes the least $\sigma$-algebra generated by $\{B(s)-B(\tau), t \leq \tau<s<v\}$.

$C_{n}$ denotes the space of all $\boldsymbol{R}^{n}$-valued continuous functions defined on $[0 \infty)$, with the usual metric $\rho$,

$$
\rho(f, g)=\sum_{n=1}^{\infty} \frac{1}{2^{n}} \frac{\sup _{t \leq n}|f(t)-g(t)|}{1+\sup _{t \leq n}|f(t)-g(t)|}, \quad f, g \in C_{n}
$$

where | | means the Euclid norm of $\boldsymbol{R}^{n}$. Let $S_{t}$ be the $\sigma$-algebra generated by $\{f(s), s \leq t\}$. According to Beneš [1], we define the $\sigma$-algebra $G_{n}$ on $[0 \infty) \times C_{n}$ as follows, a Borel subset $E$ of $[0 \infty) \times C_{n}$ is in $G_{n}$, if and only if

(i) every $t$-section of $E$ is $S_{t}$-measurable, for $t \in[0 \infty)$, and

(ii) every $f$-section of $E$ is a Borel set of $[0 \infty)$, for $f \in C_{n}$. 
Let $X(t), t \geq 0$, be a $\boldsymbol{R}^{n}$-valued stochastic process with continuous simple paths. Define $\pi ;[0 \infty) \times \Omega \rightarrow[0 \infty) \times C_{n}$, by $\pi(t \omega)=(t X(\omega))$. We denote $\pi^{-1}\left(G_{n}\right)$ by $\mathbb{S S}_{n}$.

Let the control region $\Gamma$ be a compact subset of $\boldsymbol{R}^{m}$. A process $U(t), t \geq 0$, is called an admissible control if, with probability 1 ,

(i) $U(t) \in \Gamma, \quad 0 \leq t$, and if

(ii) $\mathfrak{B}_{t}(U, B)$ is independent of $\mathfrak{B}_{t \infty}(d B)$ for every $t \geq 0$.

To be more precisely $(B, U)$ is called an admissible system. We denote, by $\mathfrak{A}$, the set of all admissible systems.

Let $\alpha(t f u)$ and $\beta(t f u)$ be an $n$-vector and an $n \times n$ matrix valued $G_{n} \times \mathfrak{B}_{m}(\Gamma)$-measurable function, defined on $[0 \infty) \times C_{n} \times \Gamma$. Then the equation (1.1) can be understood as

$$
d X(t)=\beta(t X U(t)) d B(t)+\alpha(t X U(t)) d t .
$$

By a solution of (1.1), we mean a stochastic process $(\tilde{X}(t) \tilde{B}(t) \tilde{U}(t)), t$ $\geq 0$, defined on a suitable probability space (we may assume the Lebesgue space [0 1 1 ), such that

(i) $\bar{X}$ has continuous paths,

(ii) $(\tilde{B} \tilde{U})$ has the same law as the given admissible system $(B U)$,

(iii) $\mathfrak{B}_{t}(\tilde{X} \tilde{B} \tilde{U})$ is independent of $\mathfrak{B}_{t \infty}(d \tilde{B})$ for any $t \geq 0$, and, with probability 1 ,

(iv) $\quad \tilde{X}(t)=x+\int_{0}^{t} \beta(s \tilde{X} \tilde{U}(s)) d \tilde{B}(s)+\int_{0}^{t} \alpha(s \tilde{X} \tilde{U}(s)) d s, \quad$ for any $t \geq 0$.

For simplicity, we call $X$ a solution of (1.1), or a response to the control $U$.

§ 2. Existence and uniqueness of solution. Let us impose following assumptions

(A.1) $\alpha(t f u)$ and $\beta(t f u)$ are $G_{n} \times \mathfrak{B}_{m}(\Gamma)$-measurable,

(A.2) there exists a bounded measure $d M$ on $(-\infty 0]$, such that

$$
\begin{gathered}
\sum_{i}\left|\alpha_{i}(t f u)-\alpha_{i}(t g u)\right|^{2}+\sum_{i j}\left|\beta_{i j}(t f u)-\beta_{i j}(t g u)\right|^{2} \\
\leq \int_{-t}^{0}|f(s+t)-g(s+t)|^{2} d M(s)
\end{gathered}
$$

and

(A.3) there exists a increasing function $L(t)$, such that

$$
\sum_{i}\left|\alpha_{i}(t 0 u)\right|^{2}+\sum_{i j}\left|\beta_{i j}(t 0 u)\right|^{2} \leq L(t), \quad \forall u \in \Gamma .
$$


THEOREM 1. Under assumptions (A 1) (A 2) and (A 3) there exists a solution of (1.1) uniquely. Moreover, this solution $X(t)$ is $\mathfrak{B}_{t}(U B)$ measurable and has the following moments,

$$
E|X(t)|^{k} \leq K(k, T), \quad \text { for } t \leq T, \quad k=1,2 \cdots .
$$

$$
E|X(t)-X(s)|^{4} \leq K(T)\left((t-s)^{2}+(t-s)^{4}\right), \quad \text { for } t, s \leq T,
$$

where $K(k, T)$ and $K(T)$ are independent of an admissible control $U$ and increasing in $T$.

Proof. The method of proof is just a repeat of Sect. 2 of [4]. To show the existence of a solution, we shall use the well-known successive approximation. Let us define a sequence of approximate solutions $X_{n}, n=0,1, \cdots$, as follows,

$$
\begin{aligned}
X_{0}(t) & =x \\
X_{n+1}(t) & =x+\int_{0}^{t} \beta\left(s X_{n} U(s)\right) d B(s)+\int_{0}^{s} \alpha\left(s X_{n} U(s)\right) d s .
\end{aligned}
$$

Then the following inequality will be proved by induction;

$$
\sum_{i} E\left|X_{m i}(t)-X_{m-1}(t)\right|^{2} \leq \frac{\gamma_{1}(t) \gamma_{2}^{m} t^{m}(t+1)^{m}}{m !}, \quad m=1,2 \cdots
$$

where $\gamma_{2}$ is a constant determined by $\|M\|(=M(-\infty, 0])$ and $\gamma_{1}(t)$ is independent of $m$ and increasing in $t$.

Therefore, we have

$$
\begin{aligned}
& P\left(\sup _{0 \leq s \leq t}\left|X_{m i}(s)-X_{m-1}(s)\right|>2 \varepsilon\right) \\
& \quad \leq P\left(\int_{0}^{t} \mid \alpha_{i}\left(\tau X_{m-1} U(\tau)\right)-\alpha_{i}\left(\tau X_{m-2} U(\tau) \mid d \tau>\varepsilon\right)\right. \\
& \quad+P\left(\sup _{0 \leq s \leq t}\left|\sum_{j} \int_{0}^{s}\left[\beta_{i j}\left(\tau X_{m-1} U(\tau)\right)-\beta_{i j}\left(\tau X_{m-2} U(\tau)\right)\right] d B_{j}(\tau)\right|>\varepsilon\right) \\
& \quad \leq \varepsilon^{-2} t \int_{0}^{t} E\left|\alpha_{i}\left(\tau X_{m-1} U(\tau)\right)-\alpha_{i}\left(\tau X_{m-2} U(\tau)\right)\right|^{2} d \tau \\
& \quad+\varepsilon^{-2} n \sum_{j} \int_{0}^{t} E\left|\beta_{i j}\left(\tau X_{m-1} U(\tau)\right)-\beta_{i j}\left(\tau X_{m-2} U(\tau)\right)\right|^{2} d \tau \\
& \quad \leq \varepsilon^{-2} n \frac{\gamma_{1}(t)_{2}^{m-1} t^{m}(t+1)^{m}}{m !}\|M\| .
\end{aligned}
$$

Setting $\varepsilon=2^{-m}$, we get

$$
\sum P\left(\sup _{0 \leq s \leq t}\left|X_{m i}(s)-X_{m-1}(s)\right|>2^{-m+1}\right)<\infty .
$$


Therefore, by Borel-Cantelli's lemma, we see that, with probability 1,

$$
X_{m}(s)=x+\sum_{\ell=1}^{m}\left[X_{\ell}(s)-X_{\ell+1}(s)\right]
$$

converges uniformly on $[0 t]$, and so, on every bounded subinterval of $[0, \infty)$. Hence the limit process $X$ has continuous paths. Applying an usual method, it is easy to see that $X$ is a solution. In order to prove (2.1) we may assume that $k$ is even.

$$
\begin{aligned}
X_{m i}^{k}(t) \leq 3^{k-1}\left[x_{i}^{k}\right. & +\left(\int_{0}^{t} \alpha_{i}\left(\tau X_{m-1} U(\tau)\right) d \tau\right)^{k} \\
& \left.+\left(\sum_{j} \int_{0}^{t} \beta_{i j}\left(\tau X_{m-1} U(\tau)\right) d B_{j}(\tau)\right)^{k}\right] \\
\leq & 3^{k-1}\left[x_{i}^{k}+t^{k-1} \int_{0}^{t} \alpha_{i}^{k} d \tau+n^{k-1} \sum_{j}\left(\int_{0}^{t} \beta_{i j} d B_{j}\right)^{k}\right]
\end{aligned}
$$

Putting $\xi(t)=\int_{0}^{t} \beta_{i j} d B_{j}$, we shall evaluate its $k$-th moment. Let $\sigma_{A}$ be the first passage time of $\xi$ to $(-A, A)^{c}$. From a formula on stochastic differentials [6], we get

$$
E \xi_{A}^{k}(t)=\frac{k(k-1)}{2} \int_{0}^{t} E \xi_{A}^{k-2}(s) d s
$$

where $\xi_{A}(t)=\xi\left(t \wedge \sigma_{A}\right)$ and $\beta_{A}=\chi_{\left[0 \sigma_{A}\right]} \beta_{i j}$. Hence by Hölder's inequality, we get

$$
E \xi_{A}^{k}(t) \leq \frac{k(k-1)}{2} \int_{0}^{t}\left(E \xi_{A}^{k}(s)\right)^{(k-2) / k}\left(E \beta_{A}^{k}(s)\right)^{2 / k} d s
$$

Since (2.5) implies that $E \xi_{A}^{k}(t)$ is increasing in $t$, we have

$$
E \xi_{A}^{k}(t) \leq \frac{k(k-1)}{2}\left(E \xi_{A}^{k}(t)\right)^{(k-2) / k} \int_{0}^{t}\left(E \beta_{A}^{k}\right)^{2 / k} d s
$$

namely,

$$
\begin{aligned}
E \xi_{A}^{k}(t) & \leq\left(\frac{k(k-1)}{2} \int_{0}^{t}\left(E \beta_{A}^{k}(s)\right)^{2 / k} d t\right)^{k / 2} \\
& \leq K_{1}(k) t^{k / 2-1} \int_{0}^{t} E \beta_{A}^{k}(s) d s .
\end{aligned}
$$

From (A 2) and (A 3), 


$$
\begin{aligned}
\left|\beta_{i j}(t f u)\right|^{k} & \leq\left(L(t)+\int_{-t}^{0}|f(s+t)|^{2} d M(s)\right)^{k / 2} \\
& \leq K_{2}(t, k)\left(1+\int_{-t}^{0}|f(s+t)|^{k} d M(s)\right) .
\end{aligned}
$$

Hence, setting $d_{m}(t)=\sup _{\substack{0 \leq s \leq t \\ i=1 . . n}} E X_{i}^{k}(s)$, we have

$$
E \xi^{k}(t) \leq \lim _{A \rightarrow \infty} E \xi_{A}^{k}(t) \leq K_{3}(k, t)\left(1+\int_{0}^{t} d_{m-1}(s) d s\right) .
$$

Therefore, by virtue of (2.4), we can easily see

$$
E X_{m i}^{k}(t) \leq K_{4}(k, t)\left(1+\int_{0}^{t} d_{m-1}(s) d s\right) \quad i=1, \cdots n, m=1,2 \cdots .
$$

where $K_{4}(k, T)$ is independent of $U$ and increasing in $t$. So we have

$$
d_{m}(t) \leq K_{4}(k, t)\left(1+\int_{0}^{t} d_{m-1}(s) d s\right)
$$

On account of $d_{0}<\infty$, this (2.6) implies (2.1).

In order to prove (2.2) we can apply a similar calculation. Since

$$
X_{i}(t)-X_{i}(s)=\int_{s}^{t} \alpha_{i}(\tau X U(\tau)) d \tau+\sum_{j} \int^{t} \beta_{i j}(\tau X U(\tau)) d B_{j}(\tau),
$$

we have

$$
\left|X_{i}(t)-X_{i}(s)\right|^{4} \leq 3^{3}\left[(t-s)^{3} \int_{s}^{t} \alpha_{i}^{4} d \tau+n^{3} \sum_{j}\left(\int_{s}^{t} \beta_{i j} d B_{j}\right)^{4}\right] .
$$

From (A 2) and (2.1) we see

$$
E \int_{s}^{t} \alpha_{i}^{4} d \tau \leq K_{5}(T)(t-s), \quad \text { for } t s \leq T,
$$

where $K_{5}(T)$ is independent of $U$. Putting $\xi(t)=\int_{s}^{t} \beta_{i j} d B_{j}$ and using same notation as above, we have

$$
\begin{aligned}
E \xi_{A}^{4}(t) & =6 \int_{s}^{t} E \xi_{A}^{2}(\tau) \beta_{A}^{2}(\tau) d \tau \\
& \leq \frac{1}{2(t-s)} \int_{s}^{t} E \xi_{A}^{4}(\tau) d \tau+18(t-s) \int_{s}^{t} E \beta_{A}^{4}(\tau) d \tau .
\end{aligned}
$$

Since $E \xi_{A}^{4}(t)$ is increasing in $t$, we get

$$
E \xi_{A}^{4}(t) \leq 36(t-s) \int_{s}^{t} E \beta_{A}^{4} d \tau .
$$


Hence from (A 2) and (2.1), we have

$$
E \xi^{4}(t) \leq \lim _{A \rightarrow \infty} E \xi_{A}^{4}(t) \leq K_{6}(T)(t-s)^{2} .
$$

Therefore (2.2) holds.

Let $\mathrm{Y}$ be a solution with bounded second moment. Recalling that $X(t)$ is $\mathfrak{B}_{t}(U, B)$-measurable, we shall evaluate $Y(t)-X(t)$. In a routine, we can show

$$
E\left|X_{i}(t)-Y_{i}(t)\right|^{2}=0, \quad i=1 \cdots n .
$$

This completes the proof of Theorem 1 .

$\S 3$. Existence of optimal controls for $\beta(t f)$. Let us introduce following assumptions

(A 4) $\beta(t f u)=\beta(t f)$

(A 5) $\beta(t f u)$ is continuous in $(t f u)$

(A 6) $\alpha(t f u)$ is continuous in (tfu)

(A 7) $\alpha(t f \Gamma)$ is convex, for each $(t f)$.

For an admissible system $(B U)$, we denote a solution of (1.1) by $X^{U}$.

THEOREM 2. Let $\Phi$ be lower semi-continuous on $C_{n}$, with $0 \leq \Phi(f) \leq \infty$. Then, under the assumptions (A 1) (A 7), there exists an admissible system $\left(U_{0} B_{0}\right)$ such that

$$
E \Phi\left(X^{U_{0}}\right) \leq E \Phi\left(X^{U}\right), \quad \forall(B U) \in \mathfrak{A}
$$

Setting $\theta^{U}(t)=\int_{0}^{t} \alpha\left(s X^{U} U(s)\right) d s$ and $\mathfrak{M}=\left\{\left(X^{U}, B, \theta^{U}\right),(B, U) \in \mathfrak{R}\right\}$, we can see, from (2.2)

LEMMA 1. Under assumptions (A 1) (A 2) and (A 3), M is L-totally bounded.

Hereafter we suppose that (A 1) (A 7) hold.

LEMMA 2. Let $X$ be the solution for $(B U)$ of $\mathfrak{A}$. Then there exists $a G_{2 n}$-measurable function $v ;[0 \infty) \times C_{2 n} \rightarrow \Gamma$, such that with probability 1 ,

$$
X(t)=x+\int_{0}^{t} \beta(s X) d B(s)+\int_{0}^{t} \alpha(s X v(s X B)) d s, \quad \forall t \geq 0
$$

Proof. Let $(\Omega \Re P)$ be a probability space on which $(X B U)$ is defined. For simplicity, we may suppose that $X(t \omega)$ and $B(t \omega)$ are continuous in $t$, for all $\omega \in \Omega$. Let us define $\pi ;\left[\begin{array}{ll}0 & \infty\end{array}\right) \times \Omega \rightarrow\left[\begin{array}{ll}0 & \infty\end{array}\right) \times C_{2 n}$ by $\pi(t \omega)$ $=(t X(\omega) B(\omega))$ and endow the $\sigma$-algebra $\mathbb{S}=\pi^{-1}\left(G_{2 n}\right)$ on $\left[\begin{array}{ll}0 & \infty\end{array}\right) \times \Omega$. 
is the completion of $\& 5$ by the product measure, Lebesque measure $\times P$. Since

$$
\theta^{U}(t)=X(t)-x-\int_{0}^{t} \beta(s \cdot X) d B(s),
$$

$\theta^{U}$ is (⿹)-measurable. Moreover $\alpha(t X U(t))$ is a (Radon-Nikodym) derivative of the right side of (3.2). So, $\alpha(t X U(t))$ is $\bar{S}$-measurable. Put $\tilde{\alpha}(t \omega u)=$ $\alpha(t X(\omega) u)$. Then $\tilde{\alpha}$ is continuous in $u$ and $\alpha(t X(\omega) U(t \omega)) \in \tilde{\alpha}(t \omega \Gamma)$. Hence an implicit function theorem [1] guarantees the existence of a $\bar{S}$-measurable $\bar{V}:[0 \infty) \times \Omega \rightarrow \Gamma$, such that

$$
\alpha(t X(\omega) U(t \omega))=\tilde{\alpha}(t \omega \bar{V}(t \omega)) .
$$

Since there exists a (B)-measurable modification $V$ of $\bar{V}$, i.e.

$$
\bar{V}(t \omega)=V(t \omega), \quad \tilde{\forall}(t \omega),
$$

we have, with probability 1 ,

$$
\theta^{U}(t \omega)=\int_{0}^{t} \alpha(s X(\omega) V(s \omega)) d s, \quad \forall t \geq 0
$$

From the definition of $\&$, $V(t \omega)$ turns out to $v(t X(\omega) B(\omega))$ with a $G_{2 n}$ measurable $v$. This completes the proof of Lemma 2 .

This lemma 2 means that we may change $U(t)$ to $v(t X B)$, if we are concerned with an event of $(X, B)$.

LEMMA 3. Suppose that $\left(X_{\ell} B_{\ell} \theta_{\ell}\right) \in \mathfrak{M}$ converges to $(X B \theta)$ in L-metric. Then $(X B \theta)$ is in $\mathfrak{M}$, i.e. there exists a control $U$ such that $(B U)$ is in $\mathfrak{U}$ and $X=X^{U}, \theta=\theta^{U}$.

Proof. By Lemma 2, we may assume

$$
X_{\ell}(t)=x+\int_{0}^{t} \beta\left(s X_{\ell}\right) d B_{\ell}(s)+\int_{0}^{t} \alpha\left(s X_{\ell} v_{\ell}\left(s X_{\ell} B_{\ell}\right)\right) d s
$$

and

$$
\theta_{\ell}(t)=\int_{0}^{t} \alpha\left(s X_{\ell} v_{\ell}\left(s X_{\ell} B_{\ell}\right)\right) d s
$$

Using Skorohod's theorem, we can construct $\left(\tilde{X}_{\ell} \tilde{B}_{\ell} \tilde{\theta}_{\ell}\right)$ and $(X B \theta)$ on the Lebesgue space (again we denote by $\Omega$ ), such that

$$
\tilde{X}_{\ell}(t)=x+\int_{0}^{t} \beta\left(s \tilde{X}_{\ell}\right) d \tilde{B}_{\ell}+\tilde{\theta}_{\ell}(t)
$$




$$
\tilde{\theta}_{\ell}(t)=\int_{0}^{t} \alpha\left(s \tilde{X}_{\ell} v_{\ell}\left(s \tilde{X}_{\ell} \tilde{B}_{\ell}\right)\right) d s
$$

and, with probability $1,\left(\tilde{X}_{\ell}(t) \tilde{B}_{\ell}(t) \tilde{\theta}_{\ell}(t)\right)$ tends to $(X(t) B(t) \theta(t))$ uniformly on any bounded subinterval of $[0 \infty)$. Hence $B$ is a Brownian process adapted to $\mathfrak{B}_{t}(X B \theta)$ and, by $(2.1)$,

$$
E|X(t)|^{4} \leq K(t)
$$

From the continuity of $\beta$, we have

$$
X(t)=x+\int_{0}^{t} \beta(s X) d B(s)+\theta(t) \quad t \geq 0 .
$$

On the other hand, by virtue of (A 2) and (A 3), we see

$$
\begin{array}{r}
|\theta(t)-\theta(s)|=\lim _{\ell}\left|\tilde{\theta}_{\ell}(t)-\tilde{\theta}_{\ell}(s)\right|=\lim _{\ell}\left|\int_{s}^{t} \alpha\left(\tau \tilde{X}_{\ell} v_{\ell}\left(\tau \tilde{X}_{\ell} \tilde{B}_{\ell}\right)\right) d \tau\right| \leq K_{1}(T \omega)|t-s| \\
\text { for } t, s \leq T .
\end{array}
$$

Moreover, setting $\alpha_{\ell}(t \omega) \equiv \alpha\left(t X(\omega) v_{\ell}\left(s \tilde{X}_{\ell}(\omega) \tilde{B}_{\ell}(\omega)\right)\right.$, we have

$$
\begin{aligned}
\mid \theta(t) & -\int_{0}^{t} \alpha_{\ell}(s) d s \mid \\
& \leq\left|\theta(t)-\tilde{\theta}_{\ell}(t)\right|+\left|\int_{0}^{t} \alpha\left(s \tilde{X}_{\ell} v_{\ell}\left(s \tilde{X}_{\ell} \tilde{B}_{\ell}\right)\right)-\alpha\left(s X v_{\ell}\left(s \tilde{X}_{\ell} \tilde{B}_{\ell}\right)\right) d s\right| \\
& \leq\left|\theta(t)-\tilde{\theta}_{\ell}(t)\right|+K_{2}(T \omega) \sup _{s \leq t}\left|\tilde{X}_{\ell}(s)-X(s)\right|, \quad t \leq T
\end{aligned}
$$

Hence, with probability 1 ,

$$
\int_{0}^{t} \alpha_{\ell}(s) d s \rightarrow \theta(t) \text {, uniformly on any bounded interval . }
$$

For simplicity, we may assume that $(X(t) B(t) \theta(t))$ is continuous in all $\omega$ and (3.4) holds for all $\omega$. Define $\pi ;[0 \infty) \times \Omega \rightarrow[0 \infty) \times C_{2 n}$ by $\pi(t \omega)=(t X(\omega) B(\omega))$ and put $\leftrightarrow s=\pi^{-1}\left(G_{2 n}\right)$. From (3.4), we can take a (s)-measurable derivative $\gamma$ of $\theta$, i.e, with probability 1 ,

$$
\theta(t)=\int_{0}^{t} \gamma(s) d s
$$

Therefore, by (3.6), we have, for any $n$-vector $\eta \in L_{2}\left(\left[\begin{array}{ll}0 & T\end{array}\right] \times \Omega\right)$,

$$
\int_{0}^{T}\left(\eta(s), \alpha_{\ell}(s)\right) d s \rightarrow \int_{0}^{T}(\eta(s), \gamma(s)) d s, \quad \tilde{\forall} \omega .
$$

Recalling (3.3), we get the following estimate, 


$$
\begin{aligned}
& E\left|\int_{0}^{T}\left(\eta(s), \alpha_{\ell}(s)\right) d s\right|^{4 / 3} \leq T^{1 / 3} E \int_{0}^{T}|\eta(s)|^{4 / 3}\left|\alpha_{\ell}(s)\right|^{4 / 3} d s \\
& \quad \leq T^{1 / 3}\left(E \int_{0}^{T}|\eta(s)|^{2} d s\right)^{2 / 3}\left(E \int_{0}^{T}\left|\alpha_{\ell}(s)\right|^{4} d s\right)^{1 / 3} \leq K_{3}(T)\|\eta\|^{4 / 3}, \quad \ell=1,2 \ldots .
\end{aligned}
$$

Therefore, by virtue of uniform integrability, $E \int_{0}^{T}\left(\eta(s), \alpha_{\ell}(s)\right) d s$ tends to $E \int_{0}^{T}(\eta(s), \gamma(s)) d s$. Consequently $\alpha_{\ell}$ tends to $\gamma$ weakly in $L_{2}\left(\left[\begin{array}{ll}0 & T\end{array}\right] \times \Omega\right)$. Hence a convex combination of $\alpha_{\ell}$ can converge to $\gamma$ strongly. So, we have a subsequence which converges almost everywhere. Since $\alpha(s X(\omega) \Gamma)$ is convex and closed, for almost all $(t \omega)$,

$$
\gamma(t \omega) \in \alpha(s X(\omega) \Gamma) .
$$

We can modify $\gamma$, so that (3.8) holds for all $(t \omega)$, i.e. there exists a $\overline{G S}-$ measurable $\bar{\gamma}$ such that

$$
\bar{\gamma}(t \omega)=\gamma(t \omega) \quad \text { for almost all }(t \omega),
$$

and

$$
\bar{\gamma}(t \omega) \in \alpha(t X(\omega) \Gamma) \quad \forall(t \omega) .
$$

Again, by an implicit function theorem, we have a $G_{2 n}$-measurable $v ;[0 \infty) \times C_{2 n} \rightarrow \Gamma$, such that

$$
\gamma(t \omega)=\alpha(t X(\omega) v(t X(\omega) B(\omega)) \quad \widetilde{\nabla}(t \omega) .
$$

Hence, $(B v(s X B))$ is an admissible system and by (3.7), with probability 1 ,

$$
\theta(t)=\int_{0}^{t} \alpha(s X v(s X B)) d s, \quad \forall t \geq 0
$$

Recalling (3.4), we conclude that $(X B \theta)$ is in $\mathfrak{M}$.

Proof of Theorem 2. Let $X_{m}$ be approximate optimal, i.e.

$$
\lim E \Phi\left(X_{m}\right)=\inf _{\mathfrak{U}} E \Phi\left(X^{U}\right) .
$$

Let $X_{m}$ be a response for $\left(B_{m} U_{m}\right)$. By Lemmas 2 and $3, \mathfrak{M}$ is sequentially compact. Hence it is enough to verify that $E \Phi(X)$ is lower semicontinuous under $L$-convergence. If $X_{m}$ tends to $X$ in $L$-metric, then Skorohod's theorem tells us that we may assume that, with probability $1, X_{m}(t)$ converges to $X(t)$ uniformly on any bounded interval. Hence, with probability 1 , 


$$
\Phi(X) \leq \underline{\lim } \Phi\left(X_{m}\right) .
$$

By Fatou's lemma, we have

$$
E \Phi(X) \leq \underline{\lim } E \Phi\left(X_{m}\right)
$$

which proves Theorem 2.

§4. Transformation of measure. Consider a stochastic differential equation

$$
d X(t)=\beta(t X) d B(t)+\gamma(t X) d t, \quad X(0)=x .
$$

We assume the following conditions,

(C 1) $\beta$ is $G_{n}$-measurable

(C 2) $\beta(t f)$ is locally square integrable in $t$, for any $f \in C_{n}$.

(C 3 ) there exists a bounded $G_{n}$-measurable $n$-vector function $\phi$, such that

$$
\gamma(t f)=\beta(t f) \phi(t f) .
$$

Under these assumptions, we can apply the method of the so-called transformation of measure [2]. We have the following

THEOREM 3. Suppose that a stochastic differential equation

$$
d \xi(t)=\beta(t \xi) d B(t), \quad \xi(0)=x
$$

has a solution and the explosion does not occur. Then (4.1) has a solution. Moreover if the law of the joint process $(\xi B)$ is unique for any solution $\xi$ of (4.2), then the law of $(X B)$ is unique for any solution $X$ of (4.1).

Proof. Put $F_{t}=\mathfrak{B}_{t}(B \xi)$ and

$$
D(t)=\exp \sum_{k=1}^{n}\left(\int_{0}^{t} \phi_{k}(s \xi) d B_{k}(s)-\frac{1}{2} \int_{0}^{t} \phi_{k}^{2}(s \xi) d s\right) .
$$

Then it is well-known that $D$ is an $F_{t}$-martingale. Define the probability measure $Q_{T}$, on $\left(\Omega, F_{T}\right)$, by

$$
d Q_{T}=D(t) d P, \quad T \geq 0 .
$$

Appealing to the extension theorem of measure, we have the probability measure $Q$ on $(\Omega F)$, where $F=\bigvee_{T} F_{T}$. The following lemma 1 is easy.

LEMMA 1. Let $\zeta$ be a bounded and $F_{t}$-measurable random variable. Then 


$$
E\left(\zeta D(t) / F_{s}\right)=\bar{E}\left(\zeta / F_{s}\right) D(s) \quad t>s,
$$

where $\bar{E}$ means the expection with respect to $Q$. Using Lemma 1, we shall show

LEMMA 2. $W(t) \equiv B(t)-\int_{0}^{t} \phi(s \xi) d s$ is a Brownian motion adapted to $F_{t}$, on $(\Omega F Q)$.

Proof. Put $Z(t) \equiv W(t) D(t)$. Then, using a formula on stochastic differentials, we have

$$
d Z(t)=D(t) d B(t)+D(t) W(t) \sum_{k=1}^{n} \phi_{k}(t \xi) d B_{k}(t)
$$

So, $Z$ is an $F_{t}$-martingale on $(\Omega F P)$. Therefore, by virtue of Lemma 1 , $W$ is an $F_{t}$-martingale on $(\Omega F P)$. Since $\bar{E}|W(t)|^{2}<\infty$, we now seek the variation process $\left\langle W_{i} W_{j}\right\rangle(t)$ on $(\Omega F Q)$, [10]. Put $Z(t) \equiv W_{i}(t) W_{j}(t) D(t)$. Then again by a formula on stochastic differentials, we have

$$
\begin{aligned}
d Z(t)= & D(t) \delta_{i j} d t+L(t)\left(W_{i}(t) d B_{j}(t)+W_{j}(t) d B_{i}(t)\right) \\
& +L(t) W_{i}(t) W_{j}(t) \sum \phi_{k}(t \xi) d B_{k}(t)
\end{aligned}
$$

Hence, from Lemma 1 , we get on $(\Omega F Q)$

$$
\left\langle W_{i} W_{j}\right\rangle(t)=\delta_{i j} t \text {. }
$$

This implies that $W$ is an $F_{t}$-Brownian motion, on $(\Omega F Q),[10]$.

Let us show that the process $\xi$ is a solution of (4.1) on $(\Omega F Q$ ). According to McKean [11], we define $\beta^{\prime}$ and $\beta^{\prime \prime}$ by

$$
\beta_{\ell}^{\prime}(t f)=2^{\ell} \int_{t-2-\ell}^{t} \beta(s f) d s \quad \text { and } \quad \beta_{\ell m}^{\prime \prime}(t f)=\beta_{\ell}^{\prime}\left(2^{-m}\left[2^{m} t\right], f\right),
$$

where $[c]$ is the largest integer less than $c$. Then $\beta^{\prime \prime}$ is $G_{n}$-measurable and simple in $t$. Moreover, by (C 2), we see that for any $f \in C_{n}$,

$$
\int_{0}^{T}\left|\beta(s f)-\beta_{\ell m}^{\prime \prime}(s f)\right|^{2} d s \rightarrow 0, \quad \text { if } m \rightarrow \infty \quad \text { and } \quad \ell \rightarrow \infty .
$$

Therefore, we can take a $G_{n}$-measurable function $\beta_{k}$, which is simple in $t$, so that

$$
P\left(\int_{0}^{T}\left|\beta(s \xi)-\beta_{k}(s \xi)\right|^{2} d s>2^{-k}\right)<2^{-k}
$$

and 


$$
Q\left(\int_{0}^{T}\left|\beta(s \xi)-\beta_{k}(s \xi)\right|^{2} d s>2^{-k}\right)<2^{-k} .
$$

Hence there exist sets $N_{i}(\in F)$, such that $P\left(N_{1}\right)=0$ and $Q\left(N_{2}\right)=0$. Moreover, for $\omega \notin N_{1}$,

$$
\int_{0}^{t} \beta_{k}(s \xi) d B(s) \rightarrow \int_{0}^{t} \beta(s \xi) d B(s), \quad \text { uniformly on }\left[\begin{array}{ll}
0 & T
\end{array}\right],
$$

and, for $\omega \notin N_{2}$

$$
\int_{0}^{t} \beta_{k}(s \xi) d W(s) \rightarrow \int_{0}^{t} \beta(s \xi) d W(s), \quad \text { uniformly on }\left[\begin{array}{ll}
0 & T
\end{array}\right] .
$$

Since $Q$ is absolutely continuous to $P$, putting $N=N_{1} \cup N_{2}$, we set that $Q(N)=0$ and (4.6) and (4.7) hold for $\omega \notin N$. Because $\beta_{k}$ is simple in $t$, we have

$$
\int_{0}^{t} \beta_{k}(s \xi) d B(s)=\int_{0}^{t} \beta_{k}(s \xi) d W(s)+\int_{0}^{t} \beta_{k}(s \xi) \phi(s \xi) d s .
$$

Furthermore,

$$
\left|\int_{0}^{t} \beta(s f) \phi(s f) d s-\int_{0}^{t} \beta_{k}(s f) \phi(s f) d s\right| \leq\|\phi\|_{\infty} \int_{0}^{t}\left|\beta(s f)-\beta_{k}(s f)\right| d s .
$$

Therefore, recalling (4.5), we see that, with $Q$-probability 1,

$$
\int_{0}^{t} \beta_{k}(s \xi) \phi(s \xi) d s \rightarrow \int_{0}^{t} \beta(s \xi) \phi(s \xi) d s, \quad \text { uniformly on }\left[\begin{array}{ll}
0 & T
\end{array}\right] \text {. }
$$

Consequently, with Q-probability 1,

$$
\xi(t)=x+\int_{0}^{t} \beta(s \xi) d W(s)+\int_{0}^{t} \gamma(s \xi) d s, \quad \forall t \geq 0 .
$$

Let $X$ be a solution of (4.1) and $\mu$ the probability law of $(X B)$. For convenience, we take the coordinate representation of $(X B)$, i.e. we endow the probability measure $\mu$ on $\Omega=C_{2 n}$, setting $X_{i}(t \omega)=\omega_{i}(t), i=$ $1 \cdots n$, and $B_{i}(t \omega)=\omega_{n+i}(t), i=1 \cdots n$. Put

$$
D(t)=\exp \left(-\sum_{k} \int_{0}^{t} \phi_{k}(s X) d B_{k}(s)-\frac{1}{2} \sum_{k} \int_{0}^{t} \phi_{k}^{2}(s X) d s\right.
$$

and $d \nu_{T}=D(T) d \mu$ on $F_{T}\left(\equiv \mathfrak{B}_{T}(X B)\right)$. Then $\nu_{T}$ can be extended to the probability measure $\nu$ on $F\left(\equiv \bigvee_{T} F_{T}\right)$ uniquely. Repeating the same calculations as (4.8), we see that, on $(\Omega F \nu), W(t) \equiv B(t)+\int_{0}^{t} \phi(s X) d s$ is an 
$F_{t}$-Brownian motion and

$$
X(t)=x+\int_{0}^{t} \beta(s X) d W(s) .
$$

Therefore the law of $(X W)$ is unique. Since $B(t)=W(t)-\int_{0}^{t} \phi(s X) d s$, the law of $(X B)$ is unique. This turns out that the law of coordinate is unique in $(\Omega F \nu)$. Hence $\nu_{T}$ is unique on $F_{T}$. This means that $\mu$ is unique on $F_{T}$, since $D(T)$ is positive. Consequently, $\mu$ is unique on $F$. This completes the proof of Theorem 3.

CORollary. Suppose that (A 8) is satisfied, besides (A 1) (A 4), (A 8) there exists a bounded $G_{n} \times \mathfrak{B}_{m}(\Gamma)$-measurable n-vector function $\phi$, such that

$$
\alpha(t f u)=\beta(t f) \phi(t f u) .
$$

Then, for any $G_{n}$-measurable function $v ;[0 \infty) \times C_{n} \rightarrow \Gamma$, the following stochastic differential equation

$$
d X(t)=\beta(t X) d B(t)+\alpha(t X v(t X)) d t, \quad X(0)=x,
$$

has a law unique solution $X$.

This means that $(B v(t X))$ is an admissible system and $X$ is the response.

5. Laws of solutions. Let $A$ be the set of all $G_{n}$-measurable functions $v ;\left[\begin{array}{ll}0 & \infty\end{array}\right) \times C_{n} \rightarrow \Gamma$. We introduce two sets of probability measures on $C_{n}$, namely

$$
\mathscr{P}=\left\{\text { law of } X^{U} ;(B U) \in \mathfrak{A}\right\}
$$

and

$$
Q=\left\{\text { law of } X^{v} ; v \in A\right\}
$$

THEOREM 4. Suppose that (A 9) is satisfied, besides (A 1) (A 4) and (A 6) ( (A 8),

(A 9) $\beta(t f u)$ is uniformly positive definite, i.e.

$$
\sum \beta_{i j}(t f u) c_{i} c_{j} \geq K|c|^{2} \quad \text { with } K>0 .
$$

Then $\mathscr{P}=Q$.

This theorem means that the response of an admissible control may be regarded as the response of control based on a complete observation. 
From Theorems 2 and 4, we can easily see

COROLLARY. Under the conditions (A 1) (A 9), there exists a function $v$ of $A$ such that $X^{v}$ is optimal, i.e.

$$
X(t)=x+\int_{0}^{t} \beta(s X) d B(s)+\int_{0}^{t} \alpha(s X v(s X)) d s
$$

and

$$
E \Phi(X)=\inf _{\mathfrak{q}} E \Phi\left(X^{U}\right)
$$

Let $X$ be the response for $(B U)$. Then we have

LEMMA 1. There exists a function $v$ of $A$, such that

$$
\left.E(\alpha(t X U)) / \mathfrak{B}_{t}(X)\right)=\alpha(t X v(t X)) \quad \tilde{\nabla}(t \omega) .
$$

Proof. Let $\phi(t \omega)$ be a measurable and $\mathfrak{B}_{t}(X)$-adapted version of the conditional expectation, $E\left(\alpha(t X U(t)) / \mathfrak{B}_{t}(X)\right)$. Hence $\phi$ is $\mathbb{S}_{n}$-measurable and, by (A 7),

$$
\phi(t \omega) \in \alpha(t X(\omega) \Gamma) \quad \tilde{\nabla}(t \omega) .
$$

Therefore we may modify $\phi$, so that (5.1) holds for any $(t \omega)$. This means that there exists a $\bar{\phi}_{n}$-measurable $\bar{\phi}$ such that $\bar{\phi}(t \omega)=\phi(t \omega)$ for almost all $(t \omega)$, and $\bar{\Xi}(t \omega) \in \alpha(t X(\omega) \Gamma)$ for all $(t \omega)$. Hence an implicit function theorem guarantees the existence of a $\overline{\mathbb{S}}_{n}$-measurable $\bar{V} ;\left[\begin{array}{ll}0 & \infty\end{array}\right)$ $\times \Omega \rightarrow \Gamma$, such that

$$
\bar{\phi}(t \omega)=\alpha(t X(\omega) \bar{V}(t \omega)) .
$$

Taking a $\mathbb{S}_{n}$-measurable modification of $\bar{V}$, we have a function $v \in A$, such that

$$
\phi(t \omega)=\alpha(t X(\omega) v(t X(\omega)) \quad \tilde{\nabla}(t \omega) .
$$

This completes the proof of Lemma 1 .

Put $Z(t)=X(t)-x-\int_{0}^{t} \alpha(s X v(s X)) d s$. Then we see

LEMMA 2. $Z$ is an $L_{2}$-martingale adapted to $\mathfrak{B}_{t}(X)$ and its variation process $\left\langle Z_{i} Z_{j}\right\rangle$ is given by

$$
\left\langle Z_{i} Z_{j}\right\rangle(t)=\sum_{\ell=1}^{n} \beta_{i \ell}(s X) \beta_{\ell j}(s X) d s .
$$

Proof. From the definition of $Z, Z(t)$ is $\mathfrak{B}_{t}(X)$-measurable. On the other hand 


$$
Z(t)=\int_{0}^{t} \beta(s X) d s+\int_{0}^{t}\left(\alpha(s X U(s))-E\left(\alpha\left(s X U(s) / \mathfrak{B}_{s}(X)\right)\right) d s\right.
$$

Hence $Z$ is an $L_{2}$-martingale adapted to $\mathfrak{B}_{t}(X)$. Using a formula on stochastic differentials, we have

$$
d\left(Z_{i}(t) Z_{j}(t)\right)=Z_{i}(t) d Z_{j}(t)+Z_{j}(t) d Z_{i}(t)+\sum_{\ell} \beta_{i \ell}(t X) \beta_{j \ell}(t X) d t .
$$

Therefore $Z_{i}(t) Z_{j}(t)-\sum_{\ell} \int_{0}^{t} \beta_{i \ell}(s X) \beta_{j \ell}(s X) d s$ is a $\mathfrak{B}_{t}(X)$-martingale. This means (5.2).

Proof of Theorem 4. In order to show $\mathscr{P} \subset Q$, we shall apply the method of the so-called innovation, [5]. Set $\theta(s f)=\beta(s f)^{-1}$. Then, by (A 9), $\theta$ is bounded, symmetric and $G_{n}$-measurable. Hence, by Lemma 2 , we can define the stochastic integral $\zeta(t)=\left(\zeta_{1}(t), \cdots, \zeta_{n}(t)\right)$,

$$
\zeta(t)=\int_{0}^{t} \theta(s X) d X(s),
$$

as an $L_{2}$-martingale adapted to $\mathfrak{B}_{t}(X)$, with

$$
\left\langle\zeta_{i} \eta\right\rangle(t)=\sum_{k} \int_{0}^{t} \theta_{i k}(s X) d\left\langle Z_{k} \eta\right\rangle(s) \quad i=1 \cdots n,
$$

for any $L_{2}$-martingale $\eta$ adapted to $\mathfrak{B}_{t}(X)$, [10]. Hence, by (5.2),

$$
\begin{aligned}
\left\langle\zeta_{j} Z_{k}\right\rangle(t) & =\sum_{\ell} \int_{0}^{t} \theta_{j \ell}(s X) d\left\langle Z_{\ell} Z_{k}\right\rangle(s) \\
& =\sum_{\ell p} \int_{0}^{t} \theta_{j \ell}(s X) \beta_{\ell p}(s X) \beta_{p k}(s X) d s .
\end{aligned}
$$

Therefore,

$$
\begin{aligned}
\left\langle\zeta_{i} \zeta_{j}\right\rangle(t) & =\sum_{k} \int_{0}^{t} \theta_{i k}(s X) d\left\langle Z_{k} \zeta_{j}\right\rangle(s) \\
& =\sum_{k \ell p} \int_{0}^{t} \theta_{i k}(s X) \theta_{j \ell}(s X) \beta_{\ell p}(s X) \beta_{p k}(s X) d s=\delta_{i j} t .
\end{aligned}
$$

This means that $\zeta$ is a Brownian process adapted to $\mathfrak{B}_{t}(X)$.

Consider the stochastic integral $\xi(t)=\int_{0}^{t} \beta(s X) d \zeta(s)$. Then

$$
\left\langle\xi_{i} \eta\right\rangle(t)=\sum_{j} \int_{0}^{t} \beta_{i j}(s X) d\left\langle\zeta_{j} \eta\right\rangle(s)
$$

Since 


$$
\left\langle\zeta_{j} \eta\right\rangle(t)=\sum_{\ell} \int_{0}^{t} \theta_{j \ell}(s X) d\left\langle Z_{\ell} \eta\right\rangle(s)
$$

we have

$$
\left\langle\zeta_{i} \eta\right\rangle(t)=\sum_{j \ell} \int_{0}^{t} \beta_{i j}(s X) \theta_{j \ell}(s X) d\left\langle Z_{\ell} \eta\right\rangle(s)=\left\langle Z_{i} \eta\right\rangle(t)
$$

This implies that, with probability $1, Z_{i}(t)=\xi_{i}(t)$ for all $t \geq 0$.

Because $\zeta$ is a Brownian motion, Cor. of Theorem 3 implies " $\mathscr{P} \subset Q$ ". Appealing to “ $\mathscr{P} \supset Q$ ", this completes the proof of Theorem 4 .

§ 6. Optimal controls for $\beta(t f u)$. In this section we drop the condition (A 4). An optimal control is obtained by a little different assumption, i.e. (A 9) instead of (A 5). But, since the solvability of stochastic differential equation

$$
d X(t)=\beta(t X v(t X)) d B, \quad v \in A
$$

is not yet decided, the synthesis problem is settled only in a weak sense. Hereafter we assume (B 1) and (B 2), besides (A 1) (A 3) and (A 9), (B 1) $\alpha(t f u)$ and $\beta(t f u)$ are continuous in $u$ for any $(t f)$, (B 2) $\left\{\left(\begin{array}{c}\beta^{2}(t f u) \\ \alpha(t f u)\end{array}\right) ; u \in \Gamma\right\}$ is convex,

Proposition 1. Let $B=\left(B_{1} \cdots B_{n}\right)$ be an $n$-dimensional Brownian process. Suppose that $e, \tilde{e}, \gamma$ and $\tilde{\gamma}$ are real non-anticipative processes, whose 4th moments are locally bounded, say $E|\eta(t)|^{4} \leq K^{4}(t), \eta=e, \tilde{e} \gamma, \tilde{\gamma}$. We define $\xi$ and $\tilde{\xi}$ by

$$
\xi(t)=\int_{0}^{t} e(s) d B_{i}(s)+\int_{0}^{t} \gamma(s) d s
$$

and

$$
\tilde{\xi}(t)=\int_{0}^{t} \tilde{e}(s) d B_{j}(s)+\int_{0}^{t} \tilde{\gamma}(s) d s
$$

Then, putting $\Delta_{n k}=\xi\left(\frac{k}{2^{n}}\right)-\xi\left(\frac{k-1}{2^{n}}\right)$ and $\tilde{\Delta}_{n k}=\tilde{\xi}\left(\frac{k}{2^{n}}\right)-\tilde{\xi}\left(\frac{k-1}{2^{n}}\right)$, we have

$$
P\left(\sup _{t \leq T}\left|\delta_{i j} \int_{0}^{t} e(s) \tilde{e}(s) d s-\sum_{k<t 2^{n}} \Delta_{n k} \tilde{\Delta}_{n k}\right|>2^{n / 4}\right)<C 2^{-n / 4}
$$

where a constant $C$ depends only on $T$ and $K(T)$. 
Proof. Put $\Delta_{n k}(t)=\Delta(t)=\xi(t)-\xi(c)$ where $c=(k-1) / 2^{n}$ and $\tilde{\Delta}(t)$ similarly. Then, in the same way as (2.2) of Theorem 1, we obtain, for $t<T$,

$$
E|\Delta(t)|^{4} \leq K_{1}(T)\left((t-c)^{2}+(t-c)^{4}\right)
$$

where $K_{1}(T)$ depends only on $T$ and $K(T)$. By a formula on stochastic differentials,

$$
\begin{aligned}
\Delta(t) \tilde{\Delta}(t)= & \int_{c}^{t} e(s) \tilde{\Delta}(s) d B_{i}(s)+\int_{c}^{t} \tilde{e}(s) \Delta(s) d B_{j}(s) \\
& +\int_{c}^{t} \gamma(s) \tilde{\Delta}(s)+\tilde{\gamma}(s) \Delta(s) d s+\delta_{i j} \int_{c}^{t} e(s) \tilde{e}(s) d s .
\end{aligned}
$$

From (6.2), we have

$$
\begin{aligned}
& E\left|\int_{c}^{t} r(s) \tilde{\Delta}(s)+\tilde{\gamma}(s) \Delta(s) d s\right| \leq K(T) \int_{c}^{t} \sqrt{E \tilde{\Delta}(s)^{2}}+\sqrt{E \Delta(s)^{2}} d s \\
& \quad \leq K_{2}(T) \int_{c}^{t} \sqrt{s-c} d s=\frac{2}{3} K_{2}(T)(t-c)^{3 / 2} \cdot \\
& E\left(\int_{c}^{t} e(s) \tilde{\Delta}(s) d B_{i}\right)^{2}=\int_{c}^{t} E e^{2}(s) \tilde{\Delta}\left(s^{2}\right) d s \leq K^{2}(T) \int_{c}^{t} \sqrt{E \tilde{\Delta}(s)^{4}} d s \\
& \quad \leq K_{3}(T) \int_{c}^{t}(s-c) d s=\frac{1}{2} K_{3}(T)(t-c)^{2} .
\end{aligned}
$$

Hence, by a martingale inequality, we see

$$
\begin{aligned}
& P\left(\sup _{t \leq T}\left|\sum_{k<t 2^{n}} \int_{(k-1) / 2^{n}}^{k / 2^{n}} e(s) \tilde{\Delta}_{n k}(s) d B_{i}(s)\right|>2^{-n / 4}\right) \\
& \quad \leq \sum_{k<T 2^{n}} E\left(\int_{(k-1) / 2^{2 n}}^{k / 2} e(s) \tilde{\Delta}_{n k}(s) d B_{i}(s)\right)^{2} 2^{n / 2}=0\left(2^{-n / 2}\right)
\end{aligned}
$$

Using (6.3) (6.4) and (6.5) we can obtain (6.1).

By virtue of Borel-Cantelli's lemma, (6.1) implies that, with probability 1 ,

$$
\lim _{n \rightarrow \infty} \sum_{k<t 2 n} \Delta_{n k} \tilde{\Delta}_{n k}=\int_{0}^{t} e(s) \tilde{e}(s) d s
$$

uniformly

on any bounded subinterval of $[0 \infty)$. We denote the left side of (6.6) by $\int_{0}^{t} d \xi(s) d \tilde{\xi}(s)$.

COROLlaRY. Let $X$ be a response for an admissible $(B U)$. Then, with probability 1 , 


$$
\int_{0}^{t} d X_{i}(s) d X_{j}(s)=\sum_{k} \int_{0}^{t} \beta_{i k}(s X U(s)) \beta_{k j}(s X U(s)) d s, \quad \forall t .
$$

Hence, using a mapping $\pi$ with $\pi(t \omega)=(t X(\omega))$, we see that the process $Y(t)=\int_{0}^{t} \beta^{2}(s X U(s)) d s$ is $\overline{\mathbb{S}}_{n}$-measurable.

Now we shall prove the following existence theorem.

THEOREM 5. Under the assumptions (A 1) (A 3), (A 9) (B 1) and (B 2), we have an optimal control.

LEMMA 1. There exists a $G_{n}$-measurable function $v ;(0 \infty) \times C_{n} \rightarrow$ $\Gamma$, such that for almost all $(t \omega)$

$$
\beta^{2}(t X(\omega) U(t \omega))=\beta^{2}(t X(\omega) v(t X(\omega)) .
$$

Because a symmetric and positive definite root of a symmetric positive definite matrix is unique, (6.7) means that, for almost all $(t \omega)$

$$
\beta(t X(\omega) U(t \omega))=\beta(t X(\omega) v(t X(\omega)) .
$$

LEMMA 2. There exists a $G_{2 n}$-measurable $w ;[0 \infty) \times C_{2 n} \rightarrow \Gamma$, such that, for almost all $(t \omega)$

$$
\beta(t X(\omega) U(t \omega))=\beta(t X(\omega) w(t X(\omega) B(\omega))
$$

and

$$
\alpha(t X(\omega) U(t \omega))=\alpha(t X(\omega) w(t X(\omega) B(\omega))
$$

Proof. Define $\pi ;\left[\begin{array}{ll}0 & \infty\end{array}\right) \times \Omega \rightarrow C_{2 n}$ by $\pi(t \omega)=(t X(\omega) B(\omega))$. Put $Z(t)$ $=\int_{0}^{t} \alpha(s X U(s)) d s$. Then $Z(t)=X(t)-x-\int_{0}^{t} \beta(s X U(s)) d s$ is also $\overline{\mathbb{S}}_{2 n}$-measurable by Lemma 1. Therefore $\alpha(t X U(t))$ is also $\overline{\mathbb{S}}_{2 n}$-measurable as a derivative of $Z$. Put $\gamma(t \omega)=\left(\begin{array}{c}\beta^{2}(t X(m) U(t \omega)) \\ \alpha(t X(\omega) U(t \omega))\end{array}\right)$ and $\tilde{\gamma}(t \omega u)=\left(\begin{array}{c}\beta^{2}(t X(\omega) u) \\ \alpha(t X(\omega) u)\end{array}\right)$. Then $\gamma(t \omega) \in \tilde{\gamma}(t \omega \Gamma)$, and $\gamma(\cdots)$ and $\tilde{\gamma}(\cdots u)$ are $\bar{\Phi}_{2 n}$-measurable. So, we have a $\overline{\mathbb{S}}_{2 n}$-measurable $\bar{W} ;[0 \infty) \times \Omega \rightarrow \Gamma$, such that

$$
\gamma(t \omega)=\tilde{\gamma}(t \omega \bar{W}(t \omega)) .
$$

Again taking a $\mathbb{S}_{2 n}$-modification of $\bar{W}$, we get a $G_{2 n}$-measurable function $w ;[0 \infty) \times C_{2 n} \rightarrow \Gamma$, such that for almost all $(t \omega)$

$$
\gamma(t \omega)=\tilde{\gamma}(t \omega w(t X(\omega) B(\omega))) .
$$

Recalling the definitions of $\gamma$ and $\tilde{\gamma}$, we have Lemma 2 , since a symmetric 
positive definite root of $\beta^{2}$ is $\beta$.

For $(B U) \in \mathfrak{A}$, we define $\theta^{U}$ and $\Theta^{U}$ by

$$
\theta^{U}(t)=\int_{0}^{t} \alpha(s X U(s)) d s
$$

and

$$
\Theta^{U}(t)=\int_{0}^{t} \beta^{2}(s X U(s)) d s
$$

where $X=X^{U}$ is a response of $(B U)$. Put $\mathfrak{M}=\left\{\left(X^{U} \theta^{U} \Theta^{U}\right),(B U) \in \mathfrak{R}\right\}$. Then, by (2.2) of Theorem 1, we can easily see the following lemma,

LEMMA 3. $\mathfrak{M}$ is L-totally bounded.

LEMMA 4. Suppose that $\left(X_{\ell} \theta_{\ell} \Theta_{\ell}\right)$ comes from an admissible system $\left(B_{\ell} U_{\ell}\right)$ and $\left(X_{\ell} \theta_{\ell} \Theta_{\ell} B_{\ell}\right)$ converges to $(X \theta \Theta B)$ in L-metric. Then $(X \theta \Theta)$ is in $\mathfrak{M}$.

Proof. By Lemma 2, we may assume $U_{\ell}(t)=v_{\ell}\left(t X_{\ell} B_{\ell}\right)$, with a $G_{2 n}$ measurable function $v_{\ell}$. Hence, using Skorohod's theorem, we can construct $\left(\tilde{X}_{\ell} \tilde{\theta}_{\ell} \tilde{\Theta}_{\ell} \tilde{B}_{\ell}\right)$ and $(\tilde{X} \tilde{\theta} \tilde{\Theta} \tilde{B})$, so that

$$
\begin{aligned}
\tilde{X}_{\ell}(t) & =x+\int_{0}^{t} \beta\left(s \tilde{X}_{\ell} v_{\ell}\left(s \tilde{X}_{\ell} \tilde{B}_{\ell}\right)\right) d \tilde{B}_{\ell}+\int_{0}^{t} \alpha\left(s \tilde{X}_{\ell} v_{\ell}\left(s \tilde{X}_{\ell} \tilde{B}_{\ell}\right)\right) d s \\
\tilde{\theta}_{\ell}(t) & =\int_{0}^{t} \alpha\left(s \tilde{X}_{\ell} v_{\ell}\left(s \tilde{X}_{\ell} \tilde{B}_{\ell}\right)\right) d s \\
\tilde{\Theta}_{\ell}(t) & =\int_{0}^{t} \beta^{2}\left(s \tilde{X}_{\ell} v_{\ell}\left(s \tilde{X}_{\ell} \tilde{B}_{\ell}\right)\right) d s
\end{aligned}
$$

and, with probability 1, $\left(\tilde{X}_{\ell}(t) \tilde{\theta}_{\ell}(t) \tilde{\Theta}_{\ell}(t) \tilde{B}_{\ell}(t)\right)$ tends to $(\tilde{X}(t) \tilde{\theta}(t) \tilde{\Theta}(t) \tilde{B}(t))$ uniformly on any bounded interval.

$$
\text { Put } \xi_{\ell}(t)=\tilde{X}_{\ell}(t)-x-\tilde{\theta}_{\ell}(t)=\int_{0}^{t} \beta\left(s \tilde{X}_{\ell} v_{\ell}\left(s \tilde{X}_{\ell} \tilde{B}_{\ell}\right)\right) d \tilde{B}_{\ell} .
$$

Then $\xi_{\ell}$ is an $L_{2}$-martingale adapted to $\mathfrak{B}_{t}\left(\tilde{X}_{\ell} \tilde{\theta}_{\ell}\right)$. Tending $\ell$ to $\infty$, we can see that $\xi(t) \equiv \tilde{X}(t)-x-\tilde{\theta}(t)$ is an $L_{2}$-martingale adapted to $\mathfrak{B}_{t}(\tilde{X} \tilde{\theta})$. Now we shall show

$$
\tilde{\Theta}_{i j}(t)=\int_{0}^{t} d \tilde{X}_{i}(s) d \tilde{X}_{j}(s)
$$

Define $\Delta_{n k}$ by $\tilde{X}_{i}\left(k / 2^{n}\right)-\tilde{X}_{i}\left((k-1) / 2^{n}\right)$ and $\tilde{\Delta}_{n k}$ similarly for $j$.

$$
\begin{aligned}
\sup _{t \leq T} & \left|\tilde{\Theta}_{i j}(t)-\sum_{k<t 2^{n}} \Delta_{n k} \tilde{\Delta}_{n k}\right| \\
& \leq \sup _{t \leq T}\left|\tilde{\Theta}_{i j}(t)-\tilde{\Theta}_{\ell i j}(t)\right|+\sup _{t \leq T}\left|\tilde{\Theta}_{\ell i j}(t)-\sum_{k<t 2^{n}} \Delta_{n k}^{\ell} \tilde{\Delta}_{n k}^{\ell}\right|
\end{aligned}
$$




$$
+\sup _{t \leq T}\left|\sum_{k<t 2^{n}} \Delta_{n k}^{\ell} \tilde{\Delta}_{n k}^{\ell}-\Delta_{n k} \tilde{\Delta}_{n k}\right| .
$$

Recalling (6.1) of Prop. 1, we have

$$
P\left(\text { nd term }>2^{-n / 4}\right) \leq C 2^{-n / 4}, \quad \ell=1,2 \cdots .
$$

For any $n$, we can take a large $\ell_{0}(n)$ so that

$$
P\left(3 \text { rd term }>2^{-n / 4}\right)<2^{-n / 4}, \quad \text { for } \ell>\ell_{0}(n)
$$

and

$$
P\left(1 \text { st term }>2^{-n / 4}\right)<2^{-n / 4}, \quad \text { for } \ell>\ell_{0}(n) .
$$

Hence we see that, with probability $1, \sum_{k<t 2^{n}} \Delta_{n k} \tilde{\Delta}_{n k}$ tends to $\tilde{\Theta}_{i j}(t)$ uniformly on any bounded interval, namely we have (6.8). Therefore $\tilde{\Theta}$ is $\overline{\mathscr{S}}_{n}$ measurable.

$$
\begin{aligned}
\left|\tilde{\Theta}_{i j}(t)-\tilde{\Theta}_{i j}(s)\right| & =\lim _{\ell \rightarrow \infty}\left|\tilde{\Theta}_{\ell i j}(t)-\tilde{\Theta}_{\ell i j}(s)\right|=\lim \left|\int_{s}^{t} \beta_{i j}^{2}\left(\tau \tilde{X}_{\ell} v_{\ell}\right) d \tau\right| \\
& <2 \lim \int_{s}^{t} d \tau\left[L(\tau)+\int_{-\tau}^{0}\left|\tilde{X}_{\ell}(\tau+\lambda)\right|^{2} d M(\lambda)\right] \\
& =2 \int_{s}^{t} d \tau\left[L(\tau)+\int_{-\tau}^{0}|\tilde{X}(\tau+\lambda)|^{2} d M(\lambda)\right]
\end{aligned}
$$

So, there exists a symmetric $\overline{\mathbb{G}}_{n}$-measurable $H$, such that

$$
\tilde{\Theta}(t)=\int_{0}^{t} H(s) d s
$$

Moreover, by (A 9), we see, setting $V_{\ell}(t)=v_{\ell}\left(t \tilde{X}_{\ell} \tilde{B}_{\ell}\right)$,

$$
\sum_{i j}\left(\tilde{\Theta}_{i j}(t)-\tilde{\Theta}_{i j}(s)\right) c_{i} c_{j}=\lim _{\ell} \int_{s}^{t} \sum \beta_{i j}^{2}\left(\tau \tilde{X}_{\ell} V_{\ell}\right) c_{i} c_{j} d \tau \geq K|c|^{2}(t-s)
$$

Hence, we may assume that $H$ is uniformly positive definite. If necessary, we may take a $\mathbb{G}_{n}$-modification of $H$, and $\tilde{\Theta}$ may be regarded as $\mathbb{S S}_{n}$-measurable. From

$$
\xi_{i}(t) \xi_{j}(t)-\tilde{\Theta}_{i j}(t)=\lim \left(\xi_{\ell i}(t) \xi_{\ell j}(t)-\tilde{\Theta}_{\ell i j}(t)\right),
$$

we see that $\xi_{i}(t) \xi_{j}(t)-\tilde{\Theta}_{i j}(t)$ is a $\mathscr{B}_{t}(\tilde{X} \tilde{\theta})$-martingale. This means

$$
\left\langle\xi_{i} \xi_{j}\right\rangle(t)=\tilde{\Theta}_{i j}(t) \text {. }
$$

Let $\sqrt{H}$ be the symmetric positive definite root of $H$. The $\sqrt{H}$ is $\mathbb{B S}_{n}$-measurable and we can define $W$ by 


$$
W(t)=\int_{0}^{t} \Lambda(s) d \xi(s)
$$

where $\Lambda=\sqrt{H^{-1}}$, i.e. $W$ is a $\mathscr{B}_{t}(\tilde{X} \tilde{\theta})$-martingale with variation process

$$
\begin{aligned}
\left\langle W_{i} W_{j}\right\rangle(t) & =\sum_{k \ell} \int_{0}^{t} \Lambda_{i k}(s) \Lambda_{\ell j}(s) d\left\langle\xi_{k} \xi_{\ell}\right\rangle(s) \\
& =\sum_{k \ell} \int_{0}^{t} \Lambda_{i k}(s) \Lambda_{\ell j}(s) H_{k \ell}(s) d s=\delta_{i j} t .
\end{aligned}
$$

Namely, $W$ is a Brownian process. Moreover, in the same way as in $\S 5$, we have

$$
\xi(t)=\int_{0}^{t} \sqrt{H}(s) d W(s)
$$

Consequently

$$
\begin{gathered}
\tilde{X}(t)=x+\int_{0}^{t} \sqrt{H(s)} d W(s)+\tilde{\theta}(t) \\
\left|\tilde{\theta}_{i}(t)-\tilde{\theta}_{i}(s)\right|=\lim \left|\tilde{\theta}_{\ell i}(t)-\tilde{\theta}_{\ell i}(s)\right|=\lim \left|\int_{s}^{t} \alpha_{i}\left(\tau \tilde{X}_{\ell} V_{\ell}\right) d \tau\right| \\
<2 \lim \int_{s}^{t} d \tau\left(\int_{-\tau}^{0}\left|\tilde{X}_{\ell}(\tau+p)\right|^{2} d M(P)+L(\tau)\right)^{1 / 2} \\
=2 \int_{s}^{t} d \tau\left(\int_{-\tau}^{0}|X(\tau+p)|^{2} d M(p)+L(\tau)\right)^{1 / 2} .
\end{gathered}
$$

Hence there exists a Radon-Nykodym derivative of $\tilde{\theta}$. Define $\pi ;\left[\begin{array}{l}0 \\ 0\end{array}\right)$ $\times \Omega \rightarrow C_{2 n}$ by $\pi(t \omega)=(t \tilde{X}(\omega) W(\omega))$. Then (6.9) tells us that $\tilde{\theta}$ is $\mathbb{S}_{2 n^{-}}$ measurable. So,

$$
\tilde{\theta}=(t) \int_{0}^{t} h(s) d s
$$

with a $\mathbb{S}_{2 n}$-measurable $h$.

Recalling the definition of $\tilde{\theta}$ and $\tilde{\Theta}$, we see that, with probability 1 ,

$$
\tilde{\Theta}_{\ell}(t)=\int_{0}^{t} \beta^{2}\left(t \tilde{X}_{\ell} V_{\ell}\right) d s \rightarrow \int_{0}^{t} H(s) d s \quad \text { uniformly on }\left[\begin{array}{ll}
0 & T
\end{array}\right]
$$

and

$$
\tilde{\theta}_{\ell}(t)=\int_{0}^{t} \alpha\left(t \tilde{X}_{\ell} V_{\ell}\right) d s \rightarrow \int_{0}^{t} h(s) d s \quad \text { uniformly on }\left[\begin{array}{ll}
0 & T
\end{array}\right] \text {. }
$$

But, from (A 2), we see 


$$
\begin{aligned}
\mid \tilde{\Theta}_{i j}(t) & -\int_{0}^{t} \beta_{i j}^{2}\left(t \tilde{X} V_{\ell}\right) d s \mid \\
& <\left|\tilde{\Theta}_{i j}(t)-\tilde{\Theta}_{\ell i j}(t)\right|+\left|\tilde{\Theta}_{\ell i j}(t)-\int_{0}^{t} \beta_{i j}^{2}\left(t \tilde{X} V_{\ell}\right) d s\right| \\
& <\left|\tilde{\Theta}_{i j}(t)-\tilde{\Theta}_{\ell i j}(t)\right|+\int_{0}^{t} d s\left(\int_{-s}^{0}\left|\tilde{X}_{\ell}(\tau+s)-\tilde{X}(\tau+s)\right|^{2} d M(\tau)\right)^{1 / 2} K_{4}(T \omega)
\end{aligned}
$$

where $K_{4}(T \omega)$ depends on $T$ and $\sup _{\substack{t \leq T \\ \ell=1,2 \ldots}}\left|\tilde{X}_{\ell}(t)\right|$. Since $\tilde{\Theta}_{\ell}$ and $\tilde{X}_{\ell}$ converge uniformly on $\left[\begin{array}{ll}0 & T\end{array}\right]$, we have, with probability 1 ,

$$
\int_{0}^{t} \beta^{2}\left(t \tilde{X} V_{\ell}\right) d s \rightarrow \int_{0}^{t} H(s) d s \quad \text { uniformly on }\left[\begin{array}{ll}
0 & T
\end{array}\right]
$$

and

$$
\int_{0}^{t} \alpha\left(t \tilde{X} V_{\ell}\right) d s \rightarrow \int_{0}^{t} h(s) d s \quad \text { uniformly on }\left[\begin{array}{ll}
0 & T
\end{array}\right] .
$$

Put $\gamma_{\ell}(t \omega)=\left(\beta_{11}^{2}\left(t \tilde{X} V_{\ell}\right), \cdots \beta_{n n}^{2}\left(t \tilde{X} V_{\ell}\right), \alpha_{1}\left(t \tilde{X} V_{\ell}\right) \cdots \alpha_{n}\left(t \tilde{X} V_{\ell}\right)\right)$ and $\gamma(t \omega)=$ $\left(H_{11}(s), \cdots H_{n n}(s), h_{1}(s) \cdots h_{n}(s)\right)$. Then for any $\left(n^{2}+n\right)$-vector $\eta \in L_{2}([0 T]$ $\times \Omega)$,

$$
\int_{0}^{T}\left(\gamma_{\ell}(s), \eta(s)\right) d s \rightarrow \int_{0}^{T}(\gamma(s), \eta(s)) d s, \quad \text { for } \tilde{\forall} \omega .
$$

Recalling (2.1), we get the following estimate

$$
\begin{aligned}
E \mid \int_{0}^{T} & \left(\gamma_{\ell}(s),\left.\eta(s) d s\right|^{4 / 3}<T^{1 / 3} E \int_{0}^{T}|\eta(s)|^{4 / 3}\left|\gamma_{\ell}(s)\right|^{4 / 3} d s\right. \\
& <T^{1 / 3}\left(E \int_{0}^{T}|\eta(s)|^{2} d s\right)^{2 / 3}\left(E \int_{0}^{T}\left|\gamma_{\ell}(s)\right|^{4}\right)^{1 / 3} \leq K_{5}(T)\|\eta\|^{4 / 3}, \quad \ell=1,2 \ldots
\end{aligned}
$$

Therefore, by virtue of uniform integrability, we have

$$
\lim _{\ell} E \int_{0}^{T}\left(\gamma_{\ell}(s), \eta(s)\right) d s=E \int_{0}^{T}(\gamma(s), \eta(s)) d s .
$$

Consequently $\gamma_{\ell}$ tends to $\gamma$ weakly in $L_{2}\left(\left[\begin{array}{ll}0 & T\end{array}\right] \times \Omega\right)$. Hence a convex combination of $\gamma_{\ell}$ can tend to $\gamma$ strongly. Therefore we can take a subsequence which converges almost everywhere. From (B 1) and (B 2), we have, for almost all $(t \omega)$,

$$
\left(\begin{array}{l}
H(t \omega) \\
h(t \omega)
\end{array}\right) \in\left\{\left(\begin{array}{c}
\beta^{2}(t \tilde{X}(\omega) u) \\
\alpha(t \tilde{X}(\omega) u)
\end{array}\right) ; u \in \Gamma\right\} .
$$

We can modify $H$ and $h$ on a null set, so that (6.10) holds for all $(t \omega)$, 
namely there exists a $\overline{\mathfrak{S}}_{2 n}$-measurable $\bar{H}$ and $\bar{h}$ such that, for any $(t \omega)$

$$
\left.\left(\begin{array}{l}
\bar{H}(t \omega) \\
\bar{h}(t \omega)
\end{array}\right) \in\left\{\begin{array}{l}
\beta^{2}(t \tilde{X}(\omega) u \\
\alpha(t \tilde{X}(\omega) u
\end{array}\right) ; u \in \Gamma\right\}
$$

Hence, from an implicit function theorem we can take a $\overline{\mathfrak{S}}_{2 n}$-measurable $\bar{V} ;[0 \infty) \times \Omega \rightarrow \Gamma$, such that

$$
\beta^{2}(t \tilde{X}(\omega) \bar{V}(t \omega))=\bar{H}(t \omega)
$$

and

$$
\alpha(t \tilde{X}(\omega) \bar{V}(t \omega))=\bar{h}(t \omega)
$$

Taking a $\mathscr{G S}_{2 n}$-modification of $\bar{V}$, we have a $G_{2 n}$-measurable function $v$; $\left[\begin{array}{ll}0 & \infty\end{array}\right) \times C_{2 n} \rightarrow \Gamma$, such that, for almost all $(t \omega)$,

$$
H(t \omega)=\beta^{2}(t \tilde{X}(\omega) v(t \tilde{X}(\omega) W(\omega)))
$$

and

$$
h(t \omega)=\alpha(t \tilde{X}(\omega) v(t \tilde{X}(\omega) W(\omega))) .
$$

Since $\beta(t \tilde{X}(\omega) v(t \tilde{X}(\omega)))$ is the symmetric positive definite root of $H(t \omega)$, we have, from (6.9)

$$
\tilde{X}(t)=x+\int_{0}^{t} \beta(t \tilde{X} v(t \tilde{X} W)) d W(s)+\int_{0}^{t} \alpha(t \tilde{X} v(t \tilde{X} W)) d s \quad t \geq 0
$$

with probability 1. This means that $(\tilde{X} \tilde{\theta} \tilde{\Theta})$ comes from the admissible system $(W v(t \tilde{X} W))$, namely $(\tilde{X} \tilde{\theta} \tilde{\Theta})$ is in $\mathfrak{M}$.

Proof of Theorem 5. By Lemmas 3 and 4, we can apply the same method as Theorem 2. Let $X_{m}$ be approximate optimal. Since $\mathfrak{M}$ is sequential compact, we have a subsequence $X_{m_{i}}$ of $X_{m}$ which converges in $\mathfrak{M}$, say $X=\lim X_{m_{i}}$. The lower semi-continuity of $\Phi$ induces

$$
E \Phi(X) \leq \underline{\lim } E \Phi\left(X_{m_{i}}\right)
$$

This completes the proof of Theorem 5 .

Concerning synthesis problems, we have

THEOREM 6. Let $(B U)$ be an admissible system and $X$ its response i.e.

$$
X(t)=x+\int_{0}^{t} \beta(s X U(s)) d B(s)+\int_{0}^{t} \alpha(s X U(s)) d s .
$$

Under the assumption of Theorem 5, we have a $G_{n}$-measurable function $v ;\left[\begin{array}{ll}0 & \infty\end{array}\right) \times C_{n} \rightarrow \Gamma$, such that 


$$
X(t)=x+\int_{0}^{t} \beta(s X v(s X)) d \zeta(s)+\int_{0}^{t} \alpha(s X v(s X)) d s
$$

with a Brownian process $\zeta$.

Proof. By Lemma 1 of Theorem 5, $\beta(t X U(t))$ may be regarded as $\left(\mathbb{S}_{n}\right.$-measurable $\psi$. This fact guarantees the possibility of the method in $\S 5$.

Let $\phi(s)$ be a $\mathbb{B S}_{n}$-measurable version of $E\left(\alpha(s X U(s)) / \mathfrak{B}_{s}(X)\right)$ and put $Z(t)=X(t)-x-\int_{0}^{t} \phi(s) d s$. Then $Z$ is an $L_{2}$-martingale adapted to $\mathfrak{B}_{t}(X)$ and its variation process is given by

$$
\left\langle Z_{i} Z_{j}\right\rangle(t)=\sum_{k} \int_{0}^{t} \psi_{i k}(s) \psi_{k j}(s) d s .
$$

Set $\theta(s)=\psi(s)^{-1}$ and define $\zeta$ by

$$
\zeta(t)=\int_{0}^{t} \theta(s) d X(s) .
$$

Then $\zeta$ is an $L_{2}$-martingale adapted to $\mathfrak{B}_{t}(X)$ and

$$
\left\langle\zeta_{i} \zeta_{j}\right\rangle(t)=\sum_{k \ell p} \int_{0}^{t} \theta_{i \varepsilon}(s) \theta_{j k}(s) \psi_{\ell p}(s) \psi_{p k}(s) d s=\delta_{i j} t .
$$

Namely, $\zeta$ is a $\mathfrak{B}_{t}(X)$-Brownian motion and

$$
Z(t)=\int_{0}^{t} \psi(s) d \zeta(s)
$$

Hence we have

$$
X(t)=x+\int_{0}^{t} \psi(s) d \zeta(s)+\int_{0}^{t} \phi(s) d s .
$$

On the other hand, for almost almost all $(t \omega)$

$$
E\left(\beta(t X U(t)) / \mathfrak{B}_{t}(X)\right)=\beta(t X(\omega) U(t \omega))=\psi(t \omega)
$$

and

$$
E\left(\alpha(t X U(t)) / \leftrightarrow_{t}(X)\right)=\phi(t \omega) .
$$

Therefore, by (B 1) and (B 2),

$$
\left(\begin{array}{l}
\psi^{2}(t \omega) \\
\phi(t \omega)
\end{array}\right) \in\left\{\left(\begin{array}{l}
\beta^{2}(t X(\omega) u) \\
\alpha(t X(\omega) u)
\end{array}\right) ; u \in \Gamma\right\}
$$

for almost all $(t \omega)$. Taking a $\overline{\mathbb{S}}_{n}$-modification of $\psi^{2}$ and $\phi$, so that $(6.13)$ 
holds for any $(t \omega)$, we have a $\overline{\mathbb{S}}_{n}$-measurable $\bar{V} ;[0 \infty) \times \Omega \rightarrow \Gamma$, such that, for almost all $(t \omega)$,

$$
\psi^{2}(t \omega)=\beta^{2}(t X(\omega) \bar{V}(t \omega)) \text { and } \phi(t \omega)=\alpha(t X(\omega) \bar{V}(t \omega)) .
$$

Hence we can take a $G_{n}$-measurable $v ;\left[\begin{array}{ll}0 & \infty\end{array}\right) \times C_{n} \rightarrow \Gamma$ such that, for almost all $(t \omega)$,

$$
\psi^{2}(t \omega)=\beta^{2}(t X(\omega) v(t X(\omega))
$$

and

$$
\phi(t \omega)=\alpha(t X(\omega) v(t X(\omega)) .
$$

Since $\psi$ is symmetric and positive definite, we see that, for almost all $(t \omega)$,

$$
\psi(t \omega)=\beta(t X(\omega) v(t X(\omega)) .
$$

Recalling (6.11), we complete the proof.

$\S 7$. Diffusion type processes. In this section, we assume that $\beta(t f u)$ $=\sigma(f(t) u)$ and $\alpha(t f u)=\gamma(f(t) u)$. Namely, we treat a stochastic differential equation of diffusion type,

$$
d X(t)=\sigma(X(t) U(t)) d B(t)+\gamma(X(t) U(t)) d t, \quad X(0)=x .
$$

Suppose that $\sigma$ and $\gamma$ are Lipschitz continuous in $x$. Then (7.1) has a unique solution $X^{U}$. When $\Phi$ is given by an integral form, Krylov proved that an optimal control can be given by a Markovian policy, i.e. he showed the following theorem

THEOREM [9]. Let $\tau(X)$ be the hitting time of $X$ to the boundary of a bounded open set $A$. Put $\Phi(U)=\int_{0}^{\tau(X)} F(X(s) U(s)) d s$, where $X=X^{U}$. Suppose that is uniformly positive definite and $\sigma, \gamma$ and $F$ are bounded and continuous in $u$. If Bellman equation

$$
\begin{gathered}
\sup _{u \in \Gamma}\left[\frac{1}{2} \sum a_{i j}(x u) \frac{\partial^{2} v(x)}{\partial x_{i} \partial x_{j}}+\sum \gamma_{i}(x u) \frac{\partial v(x)}{\partial x_{i}}-F(x u)\right]=0, \quad \text { on } A \\
v(x)=0 \quad \text { on } \partial A
\end{gathered}
$$

where $a=\sigma^{2}$, has a continuous solution $v$ of $W_{2}$, i.e. (7.2) is satisfied for almost all $x \in A$, then inf $E \Phi(U)$ can be attained by a Markovian policy.

We shall sketch the outline of his proof. Fix measurable version 
of derivatives of $v$ arbitrary. Since $\Gamma$ is compact and coefficients are continuous in $u$, the supremum is attained. From an implicit function theorem, we take a Borel function $w ; A \rightarrow \Gamma$, such that, for almost all $x$ of $A$,

$$
\frac{1}{2} \sum a_{i j}(x w(x)) v_{i j}(x)+\sum \gamma_{i}(x w(x)) v_{i}(x)-F(x w(x))=0
$$

where $v_{i}=\partial v / \partial x_{i}$ and $v_{i j}=\partial^{2} v / \partial x_{i} \partial x_{j}$. If it is necessary, we may extend $w$ to the whole $R^{n}$. By virtue of uniform positivity of $\sigma$, the stochastic differential equation

$$
d Y(t)=\sigma(Y(t) w(Y(t)) d B(t)+\gamma(Y(t) w(Y(t))) d t, \quad Y(0)=x
$$

has a solution [7]. Moreover we have, with probability 1 ,

Lebesgue meas. $\{t ; Y(t \omega) \in N\}=0$, for any null set $N$ of $R^{n}$. This means that there exists no trouble about the ambiguity of $w$ on a null set of $A$.

From a formula on stochastic differentials [7], [8],

$$
\begin{aligned}
v(X(t))-v(x)= & \int_{0}^{t} \frac{1}{2} \sum a_{i j}(X(s) U(s)) v_{i j}(X(s))+\sum \gamma_{i}(X(s) U(s)) v_{i}(X(s)) d s \\
& + \text { martingale }
\end{aligned}
$$

Hence we have

$$
\begin{aligned}
-v(x) & =E \int_{0}^{\sigma(X)} \frac{1}{2} \sum a_{i j}(X(s) U(s)) v_{i j}(X(s))+\sum \gamma_{i}(X(s) U(s)) v_{i}(X(s)) d s \\
& <\int_{0}^{\sigma(X)} F(X(s) U(s) d s .
\end{aligned}
$$

On the other hand,

$$
-v(x)=E \int_{0}^{\sigma(Y)} F(Y(s) w(Y(s)) d s .
$$

Therefore, $Y$ is an optimal trajectory and $w$ an optimal Markovian policy.

Application to Linear Controls. Suppose that $\sigma(x u)$ and $F(x u)$ are independent of $u$ and

$$
\gamma_{i}(x u)=\sum_{j=1}^{m} \gamma_{i j}(x) u_{j}, \quad i=1 \cdots n
$$

So, we have the following Bellman equation, 


$$
\begin{gathered}
\sup _{u \in \Gamma}\left[\frac{1}{2} \sum a_{i j}(x) v_{i j}(x)+\sum v_{i}(x) \gamma_{i j}(x) u_{j}-F(x)\right]=0, \\
v=0 \quad \text { on } \partial A .
\end{gathered}
$$

If $A$ is a connected and bounded open set with a smooth boundary, all coefficients $a, \gamma$ and $F$ are bounded and smooth and $\sigma$ is uniformly positive definite, then there is a unique solution $v$ of $C(\bar{A}) \cap C^{2}(A)$, [3]. We set the inside of the parenthesis of (7.4) by $S(x u)$. Since $S(x u)$ is linear in $u$, we have

$$
\sup _{u \in \Gamma} S(x u)=\sup _{u \in \partial \Gamma} S(x u)=0, \quad x \in A
$$

namely, $S$ can be regarded as a mapping; $A \times \partial \Gamma \rightarrow R^{1}$, and by an implicit function theorem, a Borel function $w ; A \rightarrow \partial \Gamma$, such that $S(x w(x))$ $=0$, exists. Hence we get a Bang-Bang control which is an optimal Markovian policy.

When $\Phi$ is not an integral form, we have a little example, where any optimal control cannot be given by a Markovian policy.

EXAMPLE. Consider the 1-dimensional stochastic differential equation,

$$
d X(t)=d B(t)+U(t) d t, \quad X(0)=x .
$$

Let a control region $T$ be $\left[\begin{array}{ll}-1 & 1\end{array}\right]$ and $\Phi(f)=f(1) f(2)$. Hence,

$$
E \Phi(X)=E X(1) X(2)=E X^{2}(1)+E X(1) \int_{1}^{2} U(s) d s
$$

Since any non-anticipative process $U(t)$, such that $U(t)$ is in $\Gamma$, is an admissible control, we have

$$
X(1) \int_{1}^{2} U(s) d s \geq-|X(1)|
$$

and, for $U(s)=-\operatorname{sgn} X(1)$, the equality of (7.6) is satisfied. Hence, putting $v=\inf _{\mathfrak{A}} E \Phi\left(X^{U}\right)$, we see

$$
v=\inf E\left(X^{2}(1)-|X(1)|\right)=\inf E\left(|X(1)|-\frac{1}{2}\right)^{2}-\frac{1}{4} .
$$

Because the cost functional of $\left(|f(1)|-\frac{1}{2}\right)^{2}$ is non-negative and continuous, an optimal control $\tilde{U}$ exists by Theorem 2 . Since $\tilde{U}(t), t>1$, is irrelevant, the control $U_{0}$, defined by

$$
U_{0}(t)=\tilde{U}(t), \quad t \leq 1, \quad=-\operatorname{sgn} X(1) \quad t>1,
$$

is optimal, i.e. 


$$
v=E X^{U_{0}}(1) X^{U_{0}}(2)
$$

Suppose that a Markovian policy $w$ satisfies

$$
v=E X^{w}(1) X^{w}(2) \text {. }
$$

Putting $Y(t)=X^{w}(t)$, we have, from (7.6),

$$
Y(1) \int_{1}^{2} w(Y(s)) d s \geq-|Y(1)| .
$$

If the inequality of (7.7) holds with positive probability,

$$
v>E\left(|Y(1)|-\frac{1}{2}\right)^{2}-\frac{1}{4} .
$$

On the other hand, " $v \leq E\left(|Y(1)|-\frac{1}{2}\right)^{2}-\frac{1}{4}$ " is satisfied by the definition of $\tilde{U}$. Hence, we have, with probability 1 ,

$$
Y(1) \int_{1}^{2} w(Y(s)) d s=-|Y(1)| \text {. }
$$

But, $Y$ is a diffusion whose law is mutually absolutely continuous to the law of Brownian process. So, $P(Y(1)=0)=0$ and, for any Borel set $D$ with positive Lebesgue measure,

$$
p(t x D)>0 \text {. }
$$

Hence, (7.8) means that, with probability 1,

$$
\int_{1}^{2} w(Y(s)) d s=-\operatorname{sgn} Y(1) .
$$

Since $|w| \leq 1$, we have, for almost all $\omega$

$$
w(Y(s))=-\operatorname{sgn} Y(1), \quad \text { for almost all } s \text { of }[1,2]
$$

Hence, for almost all $s$ of $[1,2]$

$$
w(Y(s))=-\operatorname{sgn} Y(1), \quad \text { for almost all } \omega .
$$

Appealing to $(7.9)$, we have, $w(\cdot)=1$ for almost everywhere and $w(\cdot)$ $=-1$ for almost everywhere. This is absurd. Consequently we have not a Markovian policy which can give $v$.

\section{REFERENCES}

[1] Beneš V. E., Existence of optimal strategies based on specified informations, for a class of stochastic decision problems, SIAM J. control, 8 (1970), 179-188. 
[2] - Existence of optimal stochastic control laws, SIAM J. control, 9 (1971), 446-472.

[ 3 ] Fleming W. H., Some Markovian optimization problems, J. Math. \& Mech., 12 (1963), 131-140.

[4] Fleming W. H. \& Nisio M., On the existence of optimal stochastic controls, J. Math. \& Mech., 15 (1966), 779-794.

[ 5 ] Fujisaki M., Kallianpur G. \& Kunita H., Stochastic differential equations for the non-linear filtering problem, Osaka J. Math., 9 (1972), 19-40.

[6] Itō K., On a formula concerning stochastic differentials, Nagoya Math. J., 3 (1951), 55-65.

[ 7 ] Krylov N. V., On the stochastic integral of Itō, Th. Prob. Appl., 14 (1969), 330-336.

[8] - On an inequality in the theory of stochastic integrals, T. V., 16 (1971), 446-457.

[9] - On the control of diffusion type processes, 2nd Japan-USSR Symp. Proba. Th. (1972).

[10] Kunita H. \& Watanabe S., On square integrable martingales, Nagoya Math. J., 30 (1967), 209-245.

[11] McKean H. P., Stochastic Integrals. Acad. Press, 1969.

Kobe University 